\title{
Using Three-dimensional Convolutional Neural Networks for Alzheimer's Disease Diagnosis
}

\author{
Cheng-Jian Lin ${ }^{1,2^{*}}$ and Cheng-Wei Lin ${ }^{1}$ \\ ${ }^{1}$ Department of Computer Science and Information Engineering, \\ National Chin-Yi University of Technology, Taichung 411, Taiwan \\ ${ }^{2}$ College of Intelligence, National Taichung University of Science and Technology, Taichung 404, Taiwan
}

(Received June 30, 2021; accepted September 14, 2021)

Keywords: Alzheimer's disease, magnetic resonance imaging, convolutional neural network, uniform experimental design, transfer learning

Alzheimer's disease (AD) is an irreversible neurodegenerative disease. Pathology shows atrophy of brain tissue, senile plaques, neurofibrillary tangles, and so forth. Magnetic resonance imaging (MRI) is the most sensitive brain imaging method in a clinic, which provides detailed anatomical structure information of the brain and is commonly studied with pattern recognition methods for AD diagnosis. Most existing methods extract hand-crafted imaging features or brain region-of-interest images to train a classifier to recognize AD. In this study, a threedimensional convolutional neural network (3D-CNN) is implemented to detect AD. The proposed network is trained with 3D magnetic resonance (MR) images to extract the spatial features. A uniform experimental design is used to optimize the network parameters and improve the 3D-CNN performance. To ensure satisfactory performance on a small amount of training data, a transfer learning technology is proposed to improve the recognition rate of the 3D-CNN. In addition, the uniform experimental design (UED) method is used to determine the optimal parameter combination of the network and improve the 3D-CNN performance. The validation data in this study are from the Open Access Series of Imaging Studies (OASIS), where the OASIS-1 data set is used as the original data set and the 3D-CNN is trained as the pretraining model. Experimental results show that when 10, 30, 60, and 90\% of the OASIS-2 data set are used to train the pre-trained 3D-CNN, the average accuracy reaches $74.66,86.99,94.58$, and $97.02 \%$, respectively. In addition, compared with the original manual design parameters, the proposed 3D-CNN with the best parameter combination improves the recognition rate by 2.07 percentage points.

\section{Introduction}

The 2019 Global Dementia Report ${ }^{(1)}$ of Alzheimer's Disease International estimated that there are more than 50 million people with dementia worldwide, with most cases of dementia in low- and middle-income countries. The cost of social care of patients with dementia is very high. 
Alzheimer's disease (AD) is the most common form of dementia. It is a persistent neurological disease with symptoms that worsen over time, such as memory loss and cognitive decline. The true cause of $\mathrm{AD}$ is still unknown. However, according to most neurologists, $\mathrm{AD}$ is due to the loss of neurons and synapses in specific subcortical areas and the cerebral cortex. Losing too many blocks of neurons can even cause the tissue to shrink. When the disease is initially detected, it can be treated with drugs containing cholinesterase inhibitors and $N$-methyl-Daspartate (NMDA) receptor antagonists to slow down the abnormal aging of mental function, and antidepressants can be used to improve cognitive behavioral disorders. However, the damaged brain area cannot be fully recovered, and no effective treatment method has been developed to cure AD.

Relevant professionals diagnose dementia on the basis of clinical symptoms and brain imaging. The following are common brain imaging biomarkers: (1) computed tomography (CT): The detection of most body parts requires injection of a contrast medium. CT uses different $\mathrm{X}$-rays to penetrate the human body and obtain signals. The results of CT are combined into a cross-sectional image of a specific body part, which can be used to observe the human anatomy and the diseases of each part. (2) Positron emission tomography (PET): Substances with positrons, such as 18F-FDG, are injected into the examinee's body, and the metabolic activity of the tissue is observed through local glucose uptake. This is often used as an early diagnosis of $\mathrm{AD}$ by assessing whether there is an abnormal decrease in the brain glucose metabolism rate. (3) Magnetic resonance imaging (MRI): A magnetic resonance (MR) signal is generated by sensing the change in the magnetic field and the hydrogen atoms of the human body, and the image is calculated by a computer. MRI requires no radiation and is a non-invasive detection method with high safety. It is currently a widely used brain observation method. In MRI, the overall anatomical structure of the brain and the subtle changes in the tissue can be observed, and more detailed brain information can be obtained. Therefore, abnormal brain atrophy, hippocampal atrophy, and decreased cortical thickness in patients with dementia can be observed by MRI.

In recent years, many scholars have conducted in-depth research on the diagnosis of dementia with machine learning/deep learning approaches. Recognition methods have been developed into computer-aided systems, which are used in different types of biomarker sensing imaging to diagnose dementia, ${ }^{(2,3)}$ especially in MRI and PET examinations. Balamurugan et al. ${ }^{(4)}$ proposed a novel dimensionality-reduction-based k-nearest-neighbor classification algorithm for analyzing and classifying AD. Dinu and Ganesan ${ }^{(5)}$ introduced an instance-based k-nearest-neighbor classifier using the T-test method for joint regression and classification for early detection of AD. The experimental results showed relatively high accuracy and the method has low computational complexity compared with other methods such as ensemble random forests and probabilistic neural networks. Zhang et al. ${ }^{(6)}$ built a multi-state Markov model to investigate the significance of known risk factors from MRI scans while predicting transitions between different clinical diagnosis states. Moore et al. ${ }^{(7)}$ used a random forest to learn the relationship between pairs of data points at different time separations. Fuse et al. ${ }^{(8)}$ used Fourier descriptors to establish the shape features of specific tissues in MRI and adopted support vector machines to identify AD. Al-Khuzaie et al. ${ }^{(9)}$ divided 3D images into 2D slice images and used convolutional neural networks (CNNs) to learn these 2D images. Ullah et al. ${ }^{(10)}$ presented a CNN to detect dementia 
and $\mathrm{AD}$ in $3 \mathrm{D}$ MR images. Liu et al. ${ }^{(11)}$ adopted the image features of MRI and PET to identify $\mathrm{AD}$ using a dual-stream multimodal CNN.

Recently, many studies have used image recognition methods for analysis and obtained good recognition results. However, these feature selection methods still have certain limitations. In general, they are divided into region-of-interest (ROI)-based feature extraction methods and voxel-based feature extraction methods. The former can reduce the feature dimension and amount of calculation and can represent features with high stability. However, the fixed selection of the area may limit the feature extraction ability, making it difficult to effectively improve the recognition rate. Pan et al. ${ }^{(12)}$ used each ROI of the brain of patients with AD to train their corresponding basic classifiers and used genetic algorithms to determine the best basic classifier as an ensemble model to diagnose patients. Feng et al. ${ }^{(13)}$ performed the contourlet transformation on 90 ROIs to obtain information features in the spatial domain and diagnose AD through MRI. Voxel-based feature extraction methods can extract the feature of each voxel and have more subtle feature representation capabilities. However, feature extraction from the original highresolution images can easily lead to dimensional disasters, reducing the computational efficiency and recognition accuracy. Ji et al. ${ }^{(14)}$ used MRI to extract the voxel features of the brainstem for research and confirmed that early $\mathrm{AD}$ is caused by brainstem atrophy. Tissot et al..$^{(15)}$ used voxelbased morphometry to detect the association between plasma phosphorylation-tau181 (p-tau181) and neurodegeneration.

The above two types of feature extraction methods require the knowledge of experts in the field, which often consumes considerable human resources and time, and it is easy to overlook other abnormal tissues of AD in the brain when extracting features in the ROI. Deep learning has good research results in the fields of image recognition and medical imaging. In particular, the feature selection method is very different from the traditional feature selection method. A deep learning network can learn the inherent feature representations in data during the training process, instead of selecting features through expert knowledge in the target field. Therefore, deep learning can be applied to specific fields by non-experts, and deep features can be learned by constructing a multilayer structure. Folego et al. ${ }^{(16)}$ proposed a three-dimensional convolutional neural network (3D-CNN) model with adaptive learning to train 3D brain $\mathrm{MR}$ images, and its purpose was to design a fully automatic and fast deep learning system.

In the field of medical image recognition, it is difficult to acquire data while preserving security and patient's right to privacy. Moreover, large amounts of labor and time are required to mark data sets. Transfer learning can fine-tune a pre-trained model through a small amount of data. Therefore, in this study, we use transfer learning to solve the problem of a small amount of data in medical images. The pre-trained model retains the learned shallow image features, reduces the time cost of training from scratch, and improves the generalization ability of the deep learning model.

In the aforementioned methods, the architecture of deep learning networks is generally set by trial-and-error methods. Scientific experiments are usually conducted to explore or develop specific goals. When experimenters need to explore the affecting factors in an experiment, they use the design of experiment (DOE) method to achieve better results, shorten the development time, and reduce costs. The uniform experimental design (UED) method ${ }^{(17)}$ was proposed in 
1980, which can reduce the time cost of experiments and effectively improve the quality of results. This method has been successfully applied in various fields such as chemical engineering, pharmacy, natural science, and computer science. Zhou et al. ${ }^{(18)}$ used UED to design Xiaokeyinshui extract combinations with different formulae for treating diabetes mellitus in mice. Lin and Jeng ${ }^{(19)}$ also used UED to optimize the convolution kernel, channel number, and other parameters of deep CNNs to classify breast cancer tissue images. Similarly, UED was used in this study to optimize the parameters of the constructed 3D-CNN to improve the robustness and accuracy of the network.

In this study, we propose a 3D-CNN to detect AD. The proposed network is trained with 3D MR images to extract the spatial features. To ensure satisfactory performance on a small amount of training data, we adopt transfer learning technology to improve the recognition rate of the proposed network architecture. In addition, UED is used to optimize the network parameters and improve the 3D-CNN performance. The validation data in this study are from the Open Access Series of Imaging Studies (OASIS), which consists of both cross-sectional and longitudinal MRI data. OASIS is a series of neuroimaging data sets that are publicly available for study and analysis. Two experiments are discussed in this study. The aim of the first experiment is to verify the transfer learning on the OASIS-1 and OASIS-2 data sets. Under the same recognition target and similar data types, OASIS-1 is used as the original data set and the 3D-CNN is trained as the pre-training model. Then OASIS-2 is used to fine-tune and test the pre-trained 3D-CNN. In the second experiment, UED is used to optimize the parameters of the constructed 3D-CNN to obtain the best parameter combination. The major contributions of this study are as follows:

(1) A 3D-CNN was implemented to detect $\mathrm{AD}$ through better extraction of the spatial features from 3D MR images.

(2) A transfer learning technology was proposed to ensure satisfactory performance on a small amount of training data to improve the recognition rate of the $3 \mathrm{D}-\mathrm{CNN}$.

(3) UED was applied to determine the optimal parameter combination of the $3 \mathrm{D}-\mathrm{CNN}$ to further improve the network performance.

(4) The experimental results showed that the average accuracy of the $3 \mathrm{D}-\mathrm{CNN}$ reached $97.02 \%$; moreover, using the optimal parameter combination selected by UED, the recognition rate of the 3D-CNN was improved by up to 2.07 percentage points.

The rest of this paper is as follows. Section 2 introduces the transfer learning method and UED of the 3D-CNN. The experimental results and analysis of the transfer learning and the parameter combination of the 3D-CNN are described in Sect. 3. Section 4 gives the conclusions.

\section{Methods}

This section describes the use of the 3D-CNN to identify AD from MR images. Because MR images showing $\mathrm{AD}$ are not easy to obtain, transfer learning technology is used to verify that the database can be successfully identified in the situation of insufficient data. UED is used to improve the efficiency of designing 3D-CNN architecture parameters and the recognition rate of the network. Multiple regression analysis is applied to the experimental data to obtain the best combination of 3D-CNN parameters. 


\section{$2.1 \quad 3 D-C N N$}

Most of the past studies cut MR images into multiple slices, and then specific area features were selected through expert knowledge. Therefore, traditional feature selection methods tend to limit the feature representation. Most mature deep learning networks process 2D images and cannot directly capture the characteristics of 3D MRI. Therefore, this study uses a basic 3D-CNN and deep learning methods to learn the features of 3D images. Owing to the large amount of input calculation for 3D-CNNs and 3D images, the architecture designed in this study is relatively shallow, which can reduce the problem of overfitting in small data sets. The designed model architecture comprises two convolutional layers with a convolution kernel size of $5 \times 5 \times$ 5 and a pooling layer with a size of $2 \times 2 \times 2$. Afterwards, a $1 \times 1 \times 1$ convolutional layer is connected subsequently with an 84-node fully connected layer. Finally, the softmax calculation and a 2-node classifier are used for classification. Figure 1 illustrates the process of feature selection in the convolutional layer. A 3D convolution kernel is used to perform convolution operations on the input image and output the same 3D feature image. In the pooling layer, the dimension is also increased to three, maximum pooling is used, and the maximum output is selected from the $2 \times 2 \times 2$ 3D image voxels. That is, the feature image size and the amount of calculation are reduced. Table 1 shows the detailed network parameters.

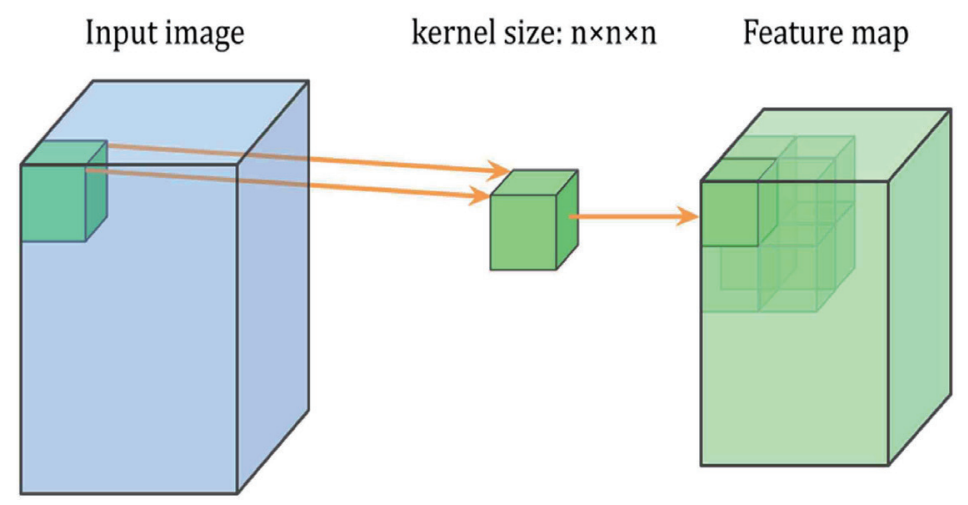

Fig. 1. (Color online) Diagram of 3D convolution operation.

Table 1

Parameters of 3D-CNN architecture.

\begin{tabular}{|c|c|c|c|c|c|}
\hline Layer & Image size & Kernel size & Stride & Padding & Filter \\
\hline Input & $95 \times 75 \times 128$ & & & & \\
\hline Convolution layer 1 & & $5 \times 5 \times 5$ & 1 & 0 & 6 \\
\hline \multicolumn{6}{|l|}{ ReLU Layer } \\
\hline MaxPooling layer 1 & & $2 \times 2 \times 2$ & 2 & & \\
\hline Convolution layer 2 & & $5 \times 5 \times 5$ & 1 & 0 & 16 \\
\hline \multicolumn{6}{|l|}{ ReLU Layer } \\
\hline MaxPooling layer 2 & & $2 \times 2 \times 2$ & 2 & & \\
\hline Convolution layer 3 & & $1 \times 1 \times 1$ & 1 & 0 & 120 \\
\hline Fully connected layer & & & & & 84 \\
\hline Fully connected layer & & & & & 2 \\
\hline
\end{tabular}




\subsection{Transfer learning}

The main purpose of transfer learning is to improve the generalization ability of the model. First, a large amount of original data are used for pre-training. The pre-training model retains the weight of the shallow network that has been trained, and then the target data set is finetuned. That is, the shallow texture features learned by the pre-trained model are used to train the target data set, and the deep features of the target data set are combined to identify the target task. This can reduce the training time from scratch. If there are two data sets, namely, Dataset-1 and Dataset-2, Dataset-1 is set as the original data set, Dataset-2 is set as the target data set, and transfer learning is performed. The constructed model is pre-trained. In this study, different proportions $(0,10,30,60$, and $90 \%)$ of the target data set (Dataset-2) are used as training data for transfer learning to verify the results.

\subsection{UED}

UED is mainly used to design experiments with multiple affecting factors and high-precision requirements. In UED, the designed experiments are evenly distributed in the overall range of experimental factors. Each factor in the experimental combination can affect the quality of the results, and a better solution can be found in the solution space while performing fewer experiments. UED is mostly used in problems with a small number of levels of each factor. If there are $s$ affecting factors in an experiment and each factor has $q$ levels, then at least $q s$ experiments must be performed. Therefore, the higher the number of levels, the greater the number of experiments. The core of UED is the design of a uniform design table. Usually, starting from the original design table, a design table with a lower deviation value is selected as the uniform design table according to the numbers of factors and levels. Because the original design table is limited and is inconvenient in practical applications, in this study, we use a good lattice point algorithm to calculate the uniform design table. The index most commonly used to measure the uniformity of a uniform design table is the centered $L_{2}$-discrepancy (centered $L_{2}$ ) [see Eq. (1)]. Through a good lattice point algorithm, the uniform design table Un(qs) with the highest uniformity is obtained. Experiments are conducted through uniform design tables, and multiple regression analysis operations are used to derive the best combination of factor levels. Figure 2 illustrates the flowchart of UED, which is divided into seven steps.

\section{Step 1. Problem definition}

The 3D-CNN parameters are manually designed on the basis of past experience. Thus, it is impossible to confirm that the selected parameters are appropriate. Therefore, we use UED to optimize the constructed 3D-CNN parameters to obtain the best combination of parameters and improve the accuracy and robustness of the network.

\section{Step 2. Determine the factor levels and the number of experiments}

In this step, we optimize the parameters used for feature extraction in the $3 \mathrm{D}$ convolutional layer to improve the feature extraction effect. To do this, we select four parameters in the first convolutional layer and the second convolutional layer of the 3D-CNN: the convolution kernel 


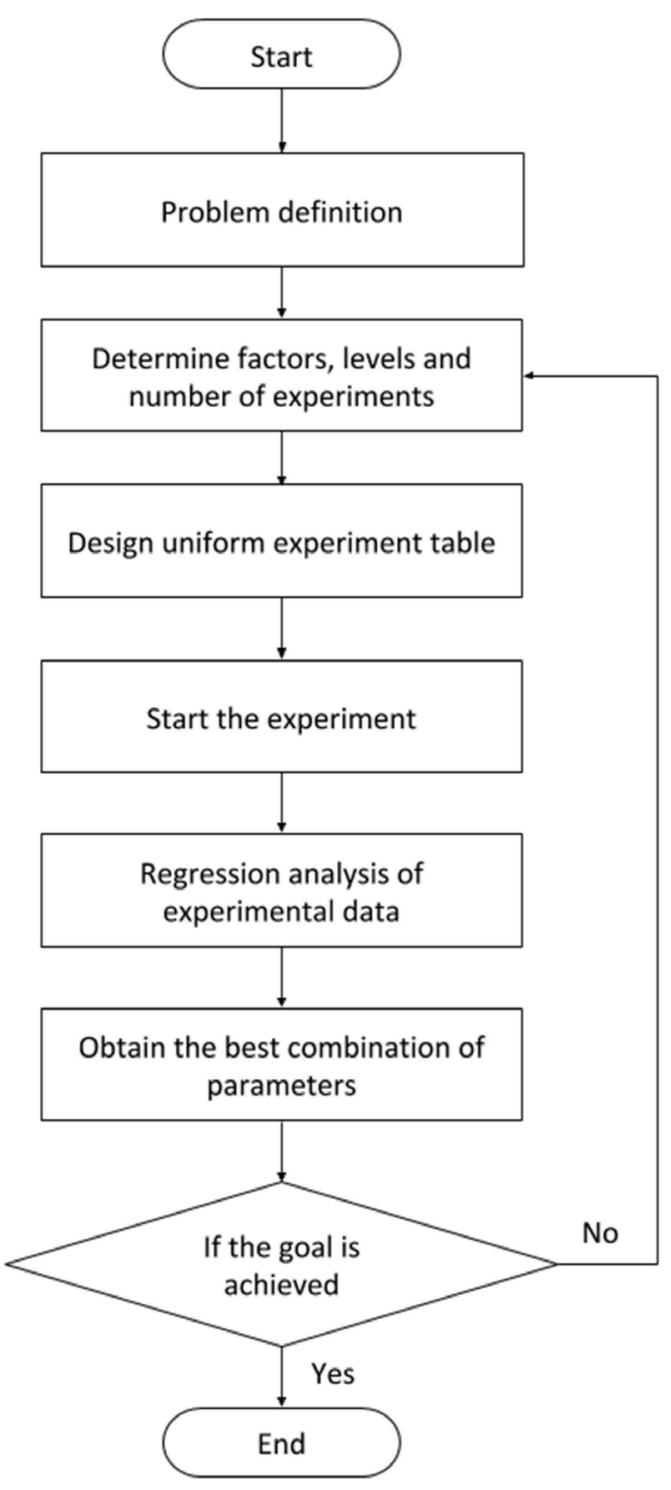

Fig. 2. Flowchart of UED.

size, the number of filters, the stride, and the padding. There are a total of eight affecting factors for the four parameters. The level of each affecting factor is designed by increasing or decreasing the size of the original 3D-CNN (Table 1). Details of the affecting factors are shown in Table 2.

\section{Step 3. Design uniform experiment table}

From the factors and levels designed in Step 2, we use the good lattice point algorithm to design the experimental table. Combining these affecting factors into experimental ranges through different levels, we select the experimental combination with the highest uniformity in the experimental range. The centralized $L_{2}$-deviation is a uniformity evaluation index, where a lower value indicates higher uniformity. Table 3 shows the obtained uniform design table. 
Table 2

Affecting factors and their levels.

\begin{tabular}{lccccc}
\hline No. & Factors & & Level 1 & Level 2 & Level 3 \\
\hline A & & Kernel size & 3 & 5 & 7 \\
B & Convolution layer 1 & Filters & 4 & 6 & 12 \\
C & Stride & 1 & 2 & \\
D & & Padding & 0 & 1 & \\
\hline E & & Kernel size & 3 & 5 & 7 \\
F & Convolution layer 2 & Filters & 8 & 16 & 32 \\
G & & Stride & 1 & 2 & \\
H & & Padding & 0 & 1 & \\
\hline
\end{tabular}

Table 3

Uniform design table with the highest uniformity.

\begin{tabular}{|c|c|c|c|c|c|c|c|c|}
\hline & \multicolumn{4}{|c|}{ Conv_1 } & \multicolumn{4}{|c|}{ Conv_2 } \\
\hline Exp. No & A & B & $\mathrm{C}$ & $\mathrm{D}$ & E & $\mathrm{F}$ & G & $\mathrm{H}$ \\
\hline 1 & 5 & 12 & 2 & 1 & 3 & 8 & 1 & 0 \\
\hline 2 & 5 & 4 & 1 & 0 & 5 & 32 & 1 & 1 \\
\hline 3 & 3 & 12 & 1 & 0 & 5 & 16 & 2 & 0 \\
\hline 4 & 3 & 4 & 1 & 1 & 3 & 32 & 1 & 0 \\
\hline 5 & 7 & 4 & 2 & 0 & 3 & 16 & 2 & 0 \\
\hline 6 & 3 & 12 & 1 & 0 & 3 & 32 & 2 & 0 \\
\hline 7 & 7 & 4 & 1 & 0 & 7 & 32 & 2 & 0 \\
\hline 8 & 7 & 6 & 1 & 1 & 3 & 16 & 1 & 1 \\
\hline 9 & 3 & 6 & 2 & 0 & 7 & 32 & 2 & 1 \\
\hline 10 & 5 & 4 & 1 & 1 & 5 & 8 & 2 & 1 \\
\hline 11 & 5 & 12 & 2 & 1 & 7 & 16 & 1 & 1 \\
\hline 12 & 5 & 4 & 2 & 0 & 7 & 16 & 2 & 0 \\
\hline 13 & 7 & 6 & 1 & 0 & 3 & 8 & 1 & 0 \\
\hline 14 & 5 & 6 & 2 & 1 & 5 & 8 & 1 & 1 \\
\hline 15 & 3 & 6 & 2 & 1 & 7 & 16 & 2 & 1 \\
\hline 16 & 7 & 6 & 1 & 1 & 5 & 8 & 1 & 1 \\
\hline 17 & 3 & 12 & 2 & 0 & 5 & 8 & 1 & 0 \\
\hline
\end{tabular}

The centralized $L_{2}$-deviation is abbreviated as $\mathrm{CD}_{2}(P)$, and its formula is as follows:

$$
\begin{aligned}
C D_{2}(P)= & \left(\frac{13}{12}\right)^{s}-\frac{2^{1-s}}{n} \sum_{k=1}^{n} \prod_{i=1}^{s}\left(2+\left|x_{k i}-\frac{1}{2}\right|-\left|x_{k i}-\frac{1}{2}\right|^{2}\right) \\
& +\frac{1}{n^{2}} \sum_{k, l=1}^{n} \prod_{i=1}^{s}\left(1+\frac{1}{2}\left|x_{k i}-\frac{1}{2}\right|+\frac{1}{2}\left|x_{l i}-\frac{1}{2}\right|-\left|x_{k i}-x_{l i}\right|\right)^{\frac{1}{2}} .
\end{aligned}
$$

Here, $n$ is the number of experiments required, $s$ is the number of affecting factors, and the smallest $n$ is obtained while satisfying $n>2 s$.

\section{Step 4. Start the experiment}

The designed experimental parameters are combined to train the 3D-CNN model, and the test accuracy is obtained. 


\section{Step 5. Regression analysis of experimental data}

Firstly, the obtained accuracy and the combination of corresponding factors $\beta_{i}$ in Step 4 are used to establish a multiple regression analysis model. Then the weights of each factor $\alpha_{1 i}, \alpha_{2 i}$, $\alpha_{3 i}$, and $\alpha_{4 i}$ are obtained through the calculation results. Finally, the weights are used to find the best parameter combination through regression analysis. The formula used in regression analysis is

$$
\varepsilon=Y-\left[\alpha_{0}+\sum_{i=1}^{n} \alpha_{1 i} \beta_{i}+\sum_{i=1}^{n} \alpha_{2 i} \beta_{i}^{2}+\sum_{i=1}^{n} \alpha_{3 i} \beta_{i}^{3}+\sum_{i=1}^{n-1} \sum_{m=i+1}^{n} \alpha_{4 i} \beta_{i} \beta_{m}\right]
$$

where $\varepsilon$ is the error, $Y$ is the accuracy, $\alpha_{0}$ is the deviation, $\alpha_{1 i}, \alpha_{2 i}, \alpha_{3 i}$, and $\alpha_{4 i}$ are the weights of $\beta$, and $\beta_{i}$ is a factor.

\section{Step 6. Obtain the best combination of parameters \\ Step 7. Confirm that the goal is achieved}

\section{Experiments and Discussion}

In this section, the effectiveness of $\mathrm{AD}$ diagnosis using the $3 \mathrm{D}-\mathrm{CNN}$ is verified. There are two experiments discussed in this study. The first is performed to verify transfer learning in two $\mathrm{AD}$ data sets, and the other uses UED to optimize the 3D-CNN parameter combination and apply it to AD diagnosis.

\subsection{Data sets}

In this study, the OASIS-1(20) and OASIS-2(21) data sets in OASIS were used as the 3D-CNN network training and testing data in the experiment, respectively. For each subject, three or four individual T1-weighted magnetization-prepared rapid gradient-echo images were acquired on a $1.5 \mathrm{~T}$ Vision scanner (Siemens, Erlangen, Germany) in a single imaging session. The OASIS-1 data set contains the categories of nondemented and demented. To build OASIS-1, a cross section of images from 416 subjects aged 18 to 96 years was collected. The OASIS-2 data set also contains the categories of nondemented and demented, but additional categories are added that correspond to different stages between nondementia and dementia. To build OASIS-2, longitudinal images from 150 subjects aged 60 to 96 years were collected.

In the two data sets, each subject underwent at least two MRI examinations with an interval of more than one year. OASIS-1 and OASIS-2 comprised 434 and 373 MRI examinations, respectively. OASIS-1 contains 316 non-AD subjects and 100 AD subjects. OASIS- 2 contains 72 non-AD subjects, $64 \mathrm{AD}$ subjects, and 14 subjects with non-AD in the initial examination but $\mathrm{AD}$ in the later examination. Tables 4 and 5 respectively show the number of images in the OASIS- 1 and OASIS-2 data sets.

In these data sets, the original size of the 3D MR images obtained is $256 \times 256 \times 128$. In the experiment, each 3D image is rescaled to $95 \times 75 \times 128$ as the input image size of the 3D-CNN on the basis of past experience. The reduction of the $3 \mathrm{D}$ images helps to reduce the burden on the 
Table 4

OASIS-1 data set.

\begin{tabular}{lcc}
\hline Class & Subjects & Number \\
\hline Nondemented & 316 & 1301 \\
Demented & 100 & 387 \\
Total & 416 & 1688 \\
\hline
\end{tabular}

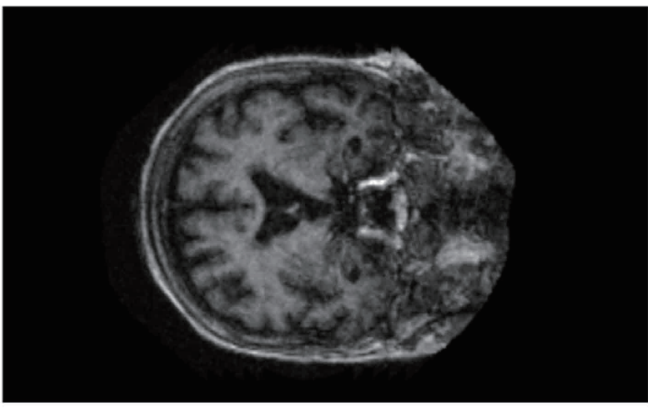

(a)
Table 5

OASIS-2 data set

\begin{tabular}{lcc}
\hline Class & Subjects & Number \\
\hline Nondemented & 72 & 692 \\
Demented & 64 & 538 \\
Total & 136 & 1220 \\
\hline
\end{tabular}

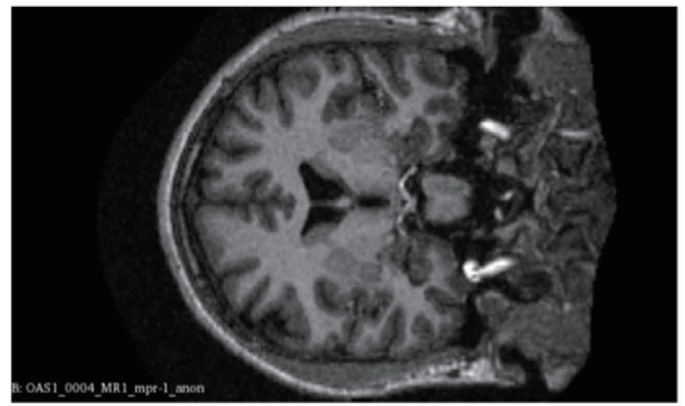

(b)

Fig. 3. MRI images in OASIS-1. (a) Demented and (b) nondemented.

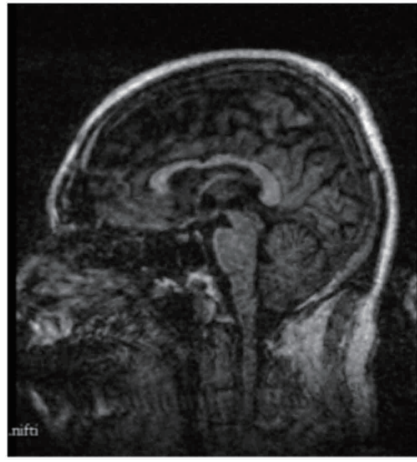

(a)

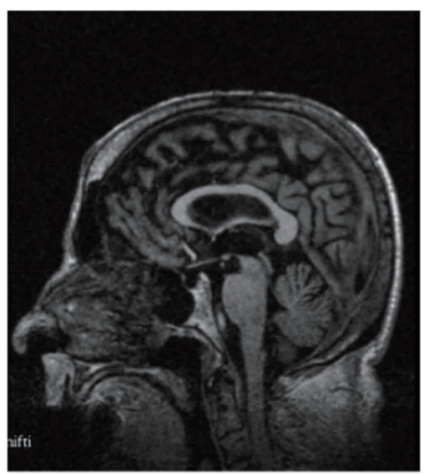

(b)

Fig. 4. MRI images in OASIS-2. (a) Demented and (b) nondemented.

hardware and speed up the calculation. Figures 3 and 4 show MR images of brains in OASIS-1 and OASIS-2, respectively.

\subsection{Experiment 1: Verification of transfer learning}

The numbers of images in OASIS-1 and OASIS-2 are 1351 and 984, respectively. Since OASIS-1 has more image data, it is selected as the original data set and OASIS-2 is used as the target data set. As mentioned in the previous subsection, the data types in the two data sets are not completely the same. OASIS-1 and OASIS-2 contain cross-sectional and longitudinal MRI data, respectively, but the target task of diagnosis is dementia/nondementia. The two data sets are used to train the 3D-CNN separately. The accuracies of the 3D-CNN trained using the OASIS- 1 and OASIS-2 data sets are 97.73 and $91.46 \%$, respectively. 
To verify the transfer learning, OASIS-1 and OASIS-2 are selected as the original data set and target data set, respectively. Table 6 shows the accuracy obtained when different proportions of data $(0,10,30,60$, and $90 \%)$ from OASIS-2 are used in the transfer learning experiment. When the pre-trained 3D-CNN using OASIS-1 directly verifies 246 data in OASIS-2, the average accuracy is $71.70 \%$. In other words, without adding part of the target data set to the training data set $(0 \%$ of training data from OASIS- $)$, the accuracy of transfer learning only reaches $70 \%$ of that of the original model for similar data types. When 10, 30, 60, and $90 \%$ of the OASIS-2 data are used to train the pre-trained 3D-CNN, the average accuracies reach 74.66 , $86.99,94.58$, and $97.02 \%$, respectively. Table 6 shows that when $60 \%$ of the OASIS- 2 data are used, the average accuracy (94.58\%) exceeds the accuracy (91.46\%) obtained when all the data in OASIS-2 are used to train a new 3D-CNN. Figure 5 shows a comparison of the accuracy and training time for different proportions of OASIS-2 data. The experiment confirmed that transfer learning between small data sets in the case of similar data (cross-sectional collection and longitudinal collection) and the same target (dementia disease and nondementia disease) maintains a high accuracy.

Table 6

Accuracy rate for different proportions of data from OASIS-2 used in the transfer learning experiment.

\begin{tabular}{lrrrcc}
\hline Data set & \multicolumn{2}{c}{ Training data } & Testing data & Avg. ACC & Avg. time \\
\hline OASIS-1 & \multicolumn{2}{c}{1351} & 337 & $97.73 \%$ & - \\
\hline & $0 \%$ & 0 & 246 & $71.70 \%$ & - \\
OASIS-2 & $10 \%$ & 98 & 246 & $74.66 \%$ & $0: 16: 56$ \\
& $30 \%$ & 295 & 246 & $86.99 \%$ & $0: 55: 59$ \\
& $60 \%$ & 590 & 246 & $94.58 \%$ & $1: 43: 59$ \\
& $90 \%$ & 886 & 246 & $97.02 \%$ & $2: 22: 27$ \\
\hline
\end{tabular}

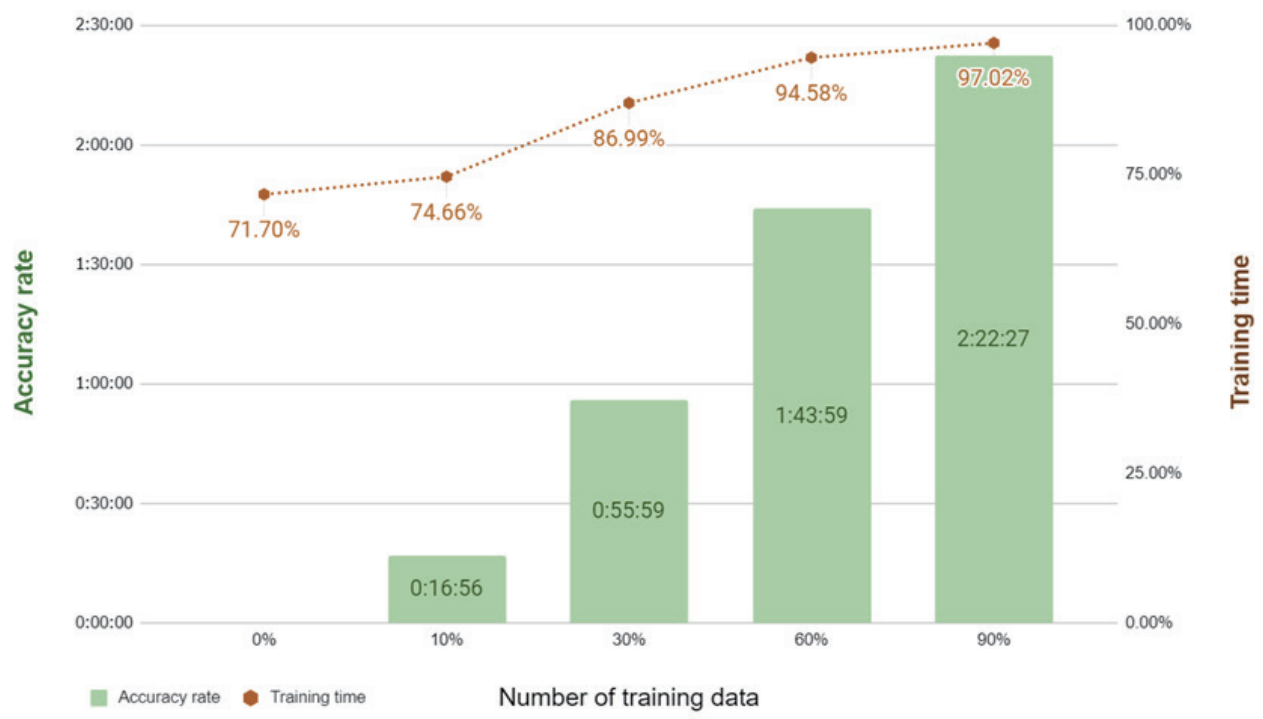

Fig. 5. (Color online) Comparison of accuracy rate and training time for different proportions of data from OASIS-2. 


\subsection{Experiment 2: Finding the best parameter combination using UED}

According to the uniform design table in Table 3, there are a total of 17 experimental combinations, and each experimental combination is composed of eight affecting factors. The parameters of the 3D-CNN are updated using these experimental combinations and the 3D-CNN is trained with OASIS- 1 to obtain the experimental results. The learning rate during training is set to 0.0001 , the maximum number of batch trainings is set to seven due to hardware computing limitations, the maximum amount of training of the overall data set is ten epochs, and stochastic gradient descent with momentum (SGDM) is added as an optimizer. The experiment is conducted on a personal computer using an NVIDIA GeForce GTX 1660 Ti graphics card with 24 GB RAM.

Each set of experiments involves three random training processes to obtain accuracies Y1, Y2, Y3, and their average accuracy Yavg. The experimental results are shown in Table 7. Among the 17 experiments, the highest average accuracy, obtained in the sixth experiment, is $99.80 \%$, and the accuracies Y1, Y2, and Y3 are 100.00, 99.70, and 99.70\%, respectively. The 3D-CNN architecture with the original parameter combination has an average accuracy of $97.73 \%$, with Y1, Y2, and Y3 of 96.74, 98.22, and 98.22\%, respectively.

After completing the experiment based on the uniform design table, we used multiple regression analysis to find the best parameter combination, as shown in Table 8. The first convolutional layer has a $5 \times 5 \times 5$ convolution kernel size, twelve filters, two strides, and one padding, and the second convolutional layer has a $3 \times 3 \times 3$ convolution kernel size, eight filters, one stride, and no padding. The accuracies of the three experimental results are 99.70, 99.70, and

Table 7

Experimental results of uniform design table.

\begin{tabular}{|c|c|c|c|c|c|c|c|c|c|c|c|c|}
\hline \multirow{2}{*}{ Run } & \multicolumn{8}{|c|}{ Factor } & \multicolumn{4}{|c|}{ Result } \\
\hline & 1 & 2 & 3 & 4 & 5 & 6 & 7 & 8 & Y1 (\%) & Y2 (\%) & Y3 (\%) & Yavg (\%) \\
\hline 1 & 5 & 12 & 2 & 1 & 3 & 8 & 1 & 0 & 99.70 & 99.70 & 99.70 & 99.70 \\
\hline 2 & 5 & 4 & 1 & 0 & 5 & 32 & 1 & 1 & 99.41 & 99.70 & 98.52 & 99.21 \\
\hline 3 & 3 & 12 & 1 & 0 & 5 & 16 & 2 & 0 & 99.11 & 99.41 & 99.70 & 99.41 \\
\hline 4 & 3 & 4 & 1 & 1 & 3 & 32 & 1 & 0 & 99.41 & 99.70 & 99.70 & 99.60 \\
\hline 5 & 7 & 4 & 2 & 0 & 3 & 16 & 2 & 0 & 98.81 & 99.11 & 99.70 & 99.21 \\
\hline 6 & 3 & 12 & 1 & 0 & 3 & 32 & 2 & 0 & 100.00 & 99.70 & 99.70 & 99.80 \\
\hline 7 & 7 & 4 & 1 & 0 & 7 & 32 & 2 & 0 & 99.70 & 99.70 & 99.41 & 99.60 \\
\hline 8 & 7 & 6 & 1 & 1 & 3 & 16 & 1 & 1 & 98.81 & 98.81 & 99.41 & 99.01 \\
\hline 9 & 3 & 6 & 2 & 0 & 7 & 32 & 2 & 1 & 99.41 & 99.11 & 99.70 & 99.41 \\
\hline 10 & 5 & 4 & 1 & 1 & 5 & 8 & 2 & 1 & 99.41 & 99.41 & 99.70 & 99.51 \\
\hline 11 & 5 & 12 & 2 & 1 & 7 & 16 & 1 & 1 & 100.00 & 99.70 & 99.41 & 99.70 \\
\hline 12 & 5 & 4 & 2 & 0 & 7 & 16 & 2 & 0 & 99.70 & 99.41 & 99.70 & 99.60 \\
\hline 13 & 7 & 6 & 1 & 0 & 3 & 8 & 1 & 0 & 99.41 & 98.81 & 99.11 & 99.11 \\
\hline 14 & 5 & 6 & 2 & 1 & 5 & 8 & 1 & 1 & 99.11 & 99.70 & 98.22 & 99.01 \\
\hline 15 & 3 & 6 & 2 & 1 & 7 & 16 & 2 & 1 & 99.70 & 99.41 & 99.41 & 99.51 \\
\hline 16 & 7 & 6 & 1 & 1 & 5 & 8 & 1 & 1 & 98.52 & 96.44 & 91.10 & 95.35 \\
\hline 17 & 3 & 12 & 2 & 0 & 5 & 8 & 1 & 0 & 99.70 & 99.70 & 99.70 & 99.70 \\
\hline
\end{tabular}


Table 8

Accuracies of the best parameter combination.

\begin{tabular}{lrrrrrrrrrrrr}
\hline Run & A & B & C & D & E & F & G & H & Exp.1 (\%) & Exp.2 (\%) & Exp.3 (\%) & Avg. (\%) \\
\hline Original & 5 & 6 & 1 & 0 & 5 & 16 & 1 & 0 & 96.74 & 98.22 & 98.22 & 97.73 \\
6th & 3 & 12 & 2 & 0 & 5 & 8 & 1 & 0 & 99.70 & 100.00 & 99.70 & 99.80 \\
UED & $\mathbf{5}$ & $\mathbf{1 2}$ & $\mathbf{2}$ & $\mathbf{1}$ & $\mathbf{3}$ & $\mathbf{8}$ & $\mathbf{1}$ & $\mathbf{0}$ & $\mathbf{9 9 . 7 0}$ & $\mathbf{9 9 . 7 0}$ & $\mathbf{1 0 0 . 0 0}$ & $\mathbf{9 9 . 8 0}$ \\
\hline
\end{tabular}

$100.00 \%$, and the average accuracy is $99.80 \%$. Compared with the original manual design parameters, the best parameter combination improves the recognition rate by 2.07 percentage points.

Compared with the existing AD diagnosis method, although the network structure in the $3 \mathrm{D}-\mathrm{CNN}^{(10)}$ is also a 3D-CNN, this model has manually designed network parameters. The network architecture consists of six layers and has a smaller input size in the network architecture. The AlexNet pre-training model ${ }^{(22)}$ is used to segment the image into 2D images for classification.

\section{Conclusions}

In this study, a 3D-CNN with a combination of transfer leaning and an optimized parameter combination is proposed for detecting AD. The proposed network is trained with 3D MR images to extract the spatial features. To ensure satisfactory performance on a small amount of training data, a transfer learning technology is proposed to improve the recognition rate of the $3 \mathrm{D}-\mathrm{CNN}$. In addition, UED is used to optimize the parameter combination of the network and improve the 3D-CNN performance. The validation data in this study are from OASIS. Two experiments are performed in this study. The purpose of the first experiment is to verify the transfer learning of the OASIS-1 and OASIS-2 data sets. Under the same recognition target and similar data types, the OASIS-1 data set is used as the original data set and 3D-CNN is trained as the pre-training model. When $10,30,60$, and $90 \%$ of the OASIS- 2 data set are used to train this pre-trained 3D-CNN, the average accuracies reach $74.66,86.99,94.58$, and $97.02 \%$, respectively. In the second experiment, a UED is used to optimize the parameters of the constructed 3D-CNN to obtain the best parameter combination. Compared with the original manually designed parameters, the proposed best parameter combination method improves the recognition rate by 2.07 percentage points. In addition, the 3D-CNN with the proposed optimization parameter combination has a higher recognition rate than existing $\mathrm{AD}$ diagnosis methods.

The 3D-CNN requires a large amount of calculation in practical applications. In future research, how to reduce the number of weights that are learned during 3D-CNN training and reduce the amount of calculation is an important topic. In addition, a hardware implementation of a 3D-CNN architecture for the real-time detection of $\mathrm{AD}$ is another crucial issue for further research. 


\section{References}

1 Alzheimer's Disease International: https://www.alzint.org/resource/world-alzheimer-report-2019/ (accessed September 2019).

2 A. Payan and G. Montana: Proc. 4th Int. Conf. Pattern Recognit. Appl. and Methods (ICPRAM 2015) 355-362.

3 K. A. Gunawardena, R. Rajapakse, N. Kodikara, and I. U. Mudalige: Proc. 2016 16th Int. Conf. Advances in ICT for Emerging Regions (ICTer) 324-324.

4 M. Balamurugan, A. Nancy, and S. Vijaykumar: Biomed. Pharmacol. 10 (2017) 1823. https://doi.org/10.13005/ bpj/1299

5 A. J. Dinu and R. Ganesan: IJATCSE 8 (2019) 29. https://doi.org/10.30534/ijatcse/2019/0581.42019.

6 L. Zhang, C. Y. Lim, T. Maiti, Y. Li, J. Choi, A. Bozoki, and D. C. Zhu: Stat. Methods Med. Res. 28 (2019) 2801. https://doi.org/10.1177/0962280218786525

7 P. J. Moore, T. J. Lyons, J. Gallacher, for the Alzheimer's Disease Neuroimaging Initiative: PLOS One 14 (2019) e0211558. https://doi.org/10.1371/journal.pone.0211558

8 H. Fuse, K. Oishi, N. Maikusa, T. Fukami, for the Japanese Alzheimer's Disease Neuroimaging Initiative: Proc. 2018 Joint 10th Int. Conf. Soft Comput. and Intell. Syst. (SCIS) and 19th Int. Symp. Adv. Intell. Syst. (ISIS) (IEEE, 2018) 1031-1034.

9 F. E. K. Al-Khuzaie, O. Bayat, and A. D. Duru: Appl. Bionics Biomech. 2021 (2021) 9. https://doi. org/10.1155/2021/6690539

10 H. M. T. Ullah, Z. Onik, R. Islam, and D. Nandi: Proc. 2018 3rd Int. Conf. Converg. Technol. (I2CT) (IEEE, 2018) 1-3.

11 M. Liu, D. Cheng, K. Wang, and Y. Wang: Neuroinformatics 16 (2018) 295. https://doi.org/10.1007/s12021-0189370-4

12 D. Pan, C. Zou, H. Rong, and A. Zeng: J. Biomed. Eng. 38 (2021) 47. https://doi.org/10.7507/1001$\underline{5515.201911046}$

13 J. Feng, S. Zhang, and L. Chen: IEEE/ACM Trans. Comput. Biol. Bioinform. (2021) 1. https://doi.org/10.1109/ TCBB.2021.3051177

14 X. Ji, H. Wang, M. Zhu, Y. He, H. Zhang, X. Chen, W. Gao, and Y. Fu: Brain Imaging Behav. 15 (2021) 49. https://doi.org/10.1007/s11682-019-00231-3

15 C. Tissot, A. L. Benedet, J. Therriault, T. A. Pascoal, F. Z. Lussier, P. Saha-Chaudhuri, M. Chamoun, M. Savard, S. S. Mathotaarachchi, G. Bezgin, Y.-T. Wang, J. F. Arias, J. L. Rodriguez, A. Snellman, N. J. Ashton, T. K. Karikari, K. Blennow, H. Zetterberg, E. D. Villers-Sidani, P. Huot, S. Gauthier, P. Rosa-Neto, for the Alzheimer's Disease Neuroimaging Initiative: Alzheimers Res. Ther. 13 (2021) 69. https://doi.org/10.1186/ s13195-021-00802-x

16 G. Folego, M. Weiler, R. F. Casseb, R. Pires, and A. Rocha: Front. Bioeng. Biotechnol. 8 (2020) 1193. https:// doi.org/10.3389/fbioe.2020.534592

17 K.-T. Fang, D. K. J. Lin, P. Winker, and Y. Zhang: Technometrics 42 (2000) 237. https://doi.org/10.1080/004017 $\underline{06.2000 .10486045}$

18 J. Zhou, J. Pan, Z. Xiang, Q. Wang, Q. Tong, J. Fang, L. Wan, and J. Chen: Data Brief. 32 (2020) 106134. https:// doi.org/10.1016/j.dib.2020.106134

19 C. J. Lin and S. Y. Jeng: Diagnostics 10 (2020) 662. https://doi.org/10.3390/diagnostics10090662

20 D. S. Marcus, T. H. Wang, J. Parker, J. G. Csernansky, J. C. Morris, and R. L. Buckner: J. Cogn. Neurosci. 19 (2007) 1498. https:// doi.org/10.1162/jocn.2007.19.9.1498

21 D. S. Marcus, A. F. Fotenos, J. G. Csernansky, J. C. Morris, and R. L. Buckner: J. Cogn. Neurosci. 22 (2010) 2677. https://doi.org/10.1162/jocn.2009.21407

22 M. Maqsood, F. Nazir, U. Khan, F. Aadil, H. Jamal, I. Mehmood, and O.-Y Song: Sensors 19 (2019) 2645. https://doi.org/10.3390/s19112645 


\section{About the Authors}

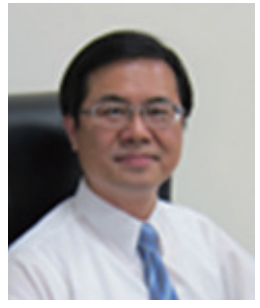

Cheng-Jian Lin received his B.S. degree in electrical engineering from Ta Tung Institute of Technology, Taipei, Taiwan, R.O.C., in 1986 and his M.S. and $\mathrm{Ph} . \mathrm{D}$. degrees in electrical and control engineering from National Chiao Tung University, Taiwan, R.O.C., in 1991 and 1996, respectively. Currently, he is a chair professor of the Computer Science and Information Engineering Department, National Chin-Yi University of Technology, Taichung, Taiwan, R.O.C., and dean of Intelligence College, National Taichung University of Science and Technology, Taichung, Taiwan, R.O.C. His current research interests are machine learning, pattern recognition, intelligent control, image processing, intelligent manufacturing, and evolutionary robots. (cjlin@ncut.edu.tw)

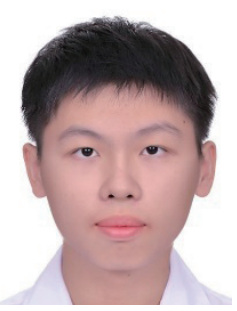

Cheng-Wei Lin received his B.S. degree from the Department of M-Commerce and Multimedia Applications, Asia University, Taichung, Taiwan, in 2020. Currently, he is a graduate student of the Department of Computer Science and Information Engineering, National Chin-Yi University of Technology, Taichung, Taiwan. His current research interests include artificial intelligence, deep learning, image recognition, and computer-aided diagnosis. (wayne39722@gmail.com) 